\section{Programas e campanhas de promoção da atividade física}

\author{
Programs and campaigns \\ to promote physical activity
}

Ma ros Santos Ferreira 1

Alberto Lopes Najar 2
${ }^{1}$ Laboratório de Atividade Física e Promoção da Saúde (LABSAU), Uerj. Rua São Francisco Xavier, 524, sala 8.133 bloco F, Maracanã, 20550-900, Rio de Ja neiro RJ. msantos@uerj.br

2 Escola Nacional de Saúde Pública.
Abstract In centive for regular physical activity has been considered an important line of action in public health, stimulating initiatives with wide population reach such as programs and campaigns aimed at promoting active lifestyles. On the present paper, we make some considerations about the pro cess of adherence to regular physical activity and the lines of action usually adopted in physical activity promotion programs (especially Agita São Paulo and Programa de Educação e Saúde através do Exercício Físico e do Esporte). We found that these programs' objectives, stra tegies and evaluation are focused on the increment of population physical activity and knowled ge of physical activity ben efits. Given the complexity of physical activity adherence, we believe that it is necessary to increase the number of variables on which actions and eval ua tion of programs should be based. In our opinion, besides the number of physically active individuals, variables such as faalities availability accessibility and quality should be considered in order to demonstrate the effectiveness of lines of action adopted, improving the design and evaluation of physical activity promotion programs with wide population reach.

Key words Physical exercise, Public policies, Adherence
Resumo O incen tivo à prática regular da atividade física vem sendo apontado como importante ação na área da saúde pública, o que vem ensejando iniciativas de larga abrangência populacional, na forma de programas e campanhas em prol de estilos de vida ativos. Neste artigo, fazemos reflex̃es sob re o processo de adesão à prática regular de atividades físicas e sobre as ações costumeiramente adotadas nesse sentido em programas de promoção da atividade física (especialmen te Agita São Paulo e Programa de Educação e Saúde através do Exercício Físico e do Esporte). Identificamos que os objetivos, estratégias e avaliações dos programas enfocam o aumento do nível de atividade física da população e a ampliação de seus conhecimentos sobre os benefícios da atividade física. Tendo em vista a complexidade da adesão à prá tica de atividades físicas, apontamos a necessidade de se ampliar o número de variáveis sobre as quais devem repousar as ações e a avaliação dos programas. A nosso ver, além do quantita tivo depessoas fisicamen te ativas, é preciso considerar variáveis como oferta, acessibilidade e qualidade de espaços para traduzir melhor o desempenho das ações adotadas, tornando mais consistentes a elaboração e a avaliação desses programas de promoção da atividade física de larga abrangência populacional.

Palavras-chave Exercício físico, Politicas públicas, Adesão 


\section{Introdução}

A necessidade de se compreen derem os condicionantes e as conseqüências da adesão ao exercício físico estende-se des de o con tex to de programas médicos supervision ados até o domínio da saúde pública. Esse campo de estudo, cujo acúmulo de conhecimen tos ainda é pequeno diante de sua importância (principalm en te no Brasil), não pode ser visto como domínio exclu sivo de uma área de conhecimen to específica.

De fato, profissionais das mais diversas áreas como psicologia, educação física, medicina, entre outras, vêm se dedicando cada dia mais ao estudo dessa temática. Apesar disso e da relativa consolidação da adesão ao exercício como área de estudos, não há mui to o que comemorar no que diz respeito à sua difusão. Com efeito, já em meados da década de 1990, Dishman (1994) apontava não ter havi do ava nços significativos no nível de prática de exercícios em nações industrializadas. Essa situação p a rece perdurar, haja vista que mais de $60 \%$ da população adulta em quase todos os países desenvo lvi dos e nas áreas urbanas do mu n domenos de senvo lvi do apres enta níveis insu ficientes de atividade física (CDC, 2000).

A com preensão e a even tual alteração desse quad ro requerem ações de larga abrangência, envolvendo profissionais de várias áreas, que tenham em comum o interesse em difundir a atividade física na pers pectiva da promoção da saúde. Nesse con tex to, in tervenções na área de políticas públicas são particularmen te importantes.

Tendo essas questões presentes, reflete-se, neste artigo, sobre o processo de adesão à ativi$\mathrm{d}$ ade física, como empreendimen to na área $\mathrm{da}$ saúde, e sobre as ações costumeiramente adotadas nesse sentido. A partir do reconhecimento da multidimensionalidade e complexidade do fenômeno da adesão, sugerimos que mais variáveis sejam consideradas na avaliação de ações em prol da prática regular de atividades físicas.

\section{Atividade física e saúde}

Qu em nunca ouviu dizer que "atividade física faz bem à saúde"? Embora expressa pelo dito popular, essa afirmação extrapola sua dimensão de senso comum. De fato, hoje, a prática regular de exercícios vem sendo apontada como im portan te ação na área de saúde pública (Sallis \& Mckenzie, 1991). Essa possibilidade encontra suporte teórico na influência benéfica da atividade física no status de saúde (Oguma et al., 2002; An ders en et al., 2000) que, por sua vez, se fundamenta na difundida associação entre exercício e indicadores de morbimortalidade que, há anos, vem sen do inve s ti gada por diferen tes autores, conforme resumido no quad ro 1 .

Contudo, apesar da associação feita entre prática regular de exercícios e saúde, é import a n te apontar que essa relação vem sen do qu e stionada. Há quem argumente, por exemplo, que ela pode ser lida de outro modo: as pessoas praticam exercícios porque gozam de melh or saúde, e não o inverso, o que caracterizaria um probl ema de "en dogen ei d ade".

Wi lliams (2003), por exemplo, a ponta limitações metodológicas nos estudos que costumam propor tal relação. $\mathrm{O}$ autor demonstrou que as conclusões obtidas por Blair et al. (1995) poderiam ser atri buídas a erro de medida na determinação dos níveis de aptidão física nas diferentes categorias. Segundo o autor, tal a rtefa to estatísti co poderia ser aplicado em outros estu dos com delineamento metodológico similar.

Solomon (1991), por sua vez, alerta sobre os peri gos da "febre" de exercícios como forma de se obter saúde e cita vários estu dos cujos resultados fragilizam a idéia de que o exercício aumenta a longevidade. Boa parte de suas argum entações é corroborada por Ca rvalho (1995), autora que critica o discurso da ativid ade física como sinônimo de saúde, o con sumismo em geral a ele associ ado e a ac riticid ade das discussões a respei to da temática.

Sobral (1990) também adverte que a associação sem reservas en tre exercício físico e saúde, numa relação de causalidade, pode levar ao campo do "otimismo ingênu o", uma vez que os benefícios do exercício dependem fundamentalmente da forma como é praticado. De fato, vá rios autores argumentam que ao de senvo lvimento da aptidão física não corresponde necessariamente uma melhoria do status de saúde (Haskell et al., 1985) e que nem todas as repercussões do exercício físico e do de s porto são benéficas à saúde (Meinberg, 1989). Há estudos, por exemplo, que demonstram que o treinamen to ao qual os atletas do de s porto de rendimen to são subm etidos pode levar, anos mais tarde, a seqüelas no organismo (Meeusen \& Borms, 1992). 


\section{Quadro 1}

Resumo dos principais estu dos que apontam associação en tre prática regular de exercícios físicos e indicadores de morbi mortalidade.

\begin{tabular}{|c|c|c|}
\hline $\begin{array}{l}\text { Morris, JN et al. } \\
\text { (1953). }\end{array}$ & $\begin{array}{l}\text { Estu do que analisou os registros de saúde de } 31.000 \\
\text { funcionários do tran sporte rodoviário (motoristas } \\
\text { e cobradores) de Londres, com idades entre } 35 \text { e } 64 \\
\text { anos, para buscar relações en tre o tipo de trabalho } \\
\text { executado e a incidência de doenças coronarianas. }\end{array}$ & $\begin{array}{l}\text { A atividade física mais intensa da atividade dos } \\
\text { cobradores foi o motivo en contrado para explicar } \\
\text { a men or incidência e mortalidade relacion adas } \\
\text { a coron a riop a tias nesse gru po. }\end{array}$ \\
\hline
\end{tabular}

Paffenbarger, RS

et al. (1970).

Paffenbarger,

RS \& Hale, AB (1975).

Paffenbarger,

RS et al. (1986).

Blair, SN et al. (1989).

Morris, JN et al. (1990).

Blair, SN et al. (1995).

Andersen, LB et al. (2000).
Es tu do lon gi tudinal que acompanhou 3.263 e s tivadores da baía de São Francisco, com o obj etivo de identificar associações entre nível de atividade física, fatores de ris co e mortalidade.

Em seqüência ao estu do anteri or, 6.351 estivadores foram acompanhados por 22 anos, até morrerem ou até complet a rem 75 anos, no que diz respei to ao nível de atividade física e mortes por doenças coronarianas.

Es tudo que examinou a prática regular de exercícios físicos e outros hábi tos de vida de 16.936 alunos e ex-alunos de Harvard, de 35 a 74 anos de idade, por um período de 16 anos.

Estu do longi tudinal em que 10.224 hom ens e 3.120 mulheres, a grupados em cinco categorias, desde sedentários até mu i to ativos, foram acompanhados por mais de oi to anos, com vistas a iden tificar os níveis de aptidão física ao risco de mortalidade.

Es tu do lon gi tudinal em que 9.376 homens, entre 45 e 64 anos e sem históri coclínico de doenças cardiovasculares, foram acompanhados durante 9 anos no tocan te aos hábi tos de vida e in tercorrências médicas.

Es tu do longi tudinal (po u co mais de 5 anos) em que 9.777 homens en tre 20 e 82 tiveram a aptidão física avaliada, com vistas a iden tificar a associação dos result ados ao risco de mortalidade.

Estu do que acompanhou 13.375 mulh eres e 17.265 homens, de 20 a 93 anos, durante 14,5 anos em média, com o obj etivo de relaci onar taxas de mortalidade (e os riscos relativos) com o nível de atividade física no lazer e no trabalho.
A atividade física pode ter maior influência no infarto do miocá rdio do que na atero sclerose.

Homens cuja atividade laboral exi gia gastos calóri cos el evados apres en ta ram men or risco para des envo lver doença coron a riana.

O estudo su gere uma associação positiva entre a prática regular de exercícios físicos e as taxas de morbid ade e mortalidade do gru po estudado.

Ap a rentemente, níveis mais altos de atividade física poster gam todo tipo de mortalidade principalmente devido a taxas mais baixas de doença cardiovascular e câncer.

O exercício físico vi goroso pode ser um fator de prevenção da doença coronariana.

Homens que mantiveram ou melhoraram aptidão física adequada apresentaram men or probabilidade de morte por todas as causas e doença card iovascular duran te o acompanhamento do que hom ens persistentemen te sedentários.

A atividade física praticada no lazer esteve inversamente associada à mortalidade por todas as causas tanto em hom ens qua $\mathrm{n}$ to em mulheres em todos os grupos etários.
Ainda que o deb a te acerca da relação entre exercício físico e saúde esteja lon ge de findar, com base no expos to até o momen to, parecenos razoável aceitar o exercício como um dos fatores que podem contribuir positivamen te $\mathrm{p}$ a ra a saúde de indiví duos e comu nid ades e, ao mesmo tem po, recusar a relação de causalidade entre eles. O fato é que a possibilidade de o exercício físico con tri buir positivam en te para a saúde vem dando suporte a iniciativas que visam engajar populações em uma vida fisica$m$ en te ativa. 


\section{A relação adesão/acesso}

A maioria dessas iniciativas tem por objetivo ampliar o conhecimen to da população sobre a prática e os benefícios dos exercícios. No entanto, se isso fosse suficiente, es pera r-se-ia que países que já vêm se debruçando sobre a problemática do sedentarismo há alguns anos, como Estados Unidos, Canadá, Grã-Bretanha e Austrália, apresentassem taxas mais animadoras de adesão ao exercício físico. Segun do King et al. (1995), a pesar das evidências dos ben efícios da prática regular de exercícios para a saúde, a maioria dos americanos permanece inativa. Esse quad ro parece ser o mesmo em muitos países industrializados, nos quais o nível de ativid ade física dos adu l tos perm a n ece bem aquém do recom en dado (CDC, 2000; Dishman, 1994). Com efeito, parece não haver evidências que su s ten tem o fato de que apenas o maior con h ecimento sobre exercício leve as pessoas a uma vida fisicamen te ativa. Segundo Dishman et al. (1985), menos de 5\% da população acredita que um maior con hecimento sobre os benefícios do exercício contribuiria para um aumento de sua prática.

A questão da motivação e adesão ao exercício é, portanto, multidimen sional (Welk, 1999; Sallis et al., 1989; Biddle, 1987; Dishman et al., 1985), o que torna complexo o estu do e estabelecimen to de diretrizes que vi semà adesão. De fato, a literatura revela que a adesão à prática de atividades físicas e desportivas sofre a influência de muitos fatores, como: ex periências a teri ores na prática desportiva e de exercícios físicos; apoio do cônjuge e de familiares (King et al., 1992); aconselhamen to médico (Bull \& Jamrozik, 1998); conveniência do local de exercitação (An d rew et al., 1981); aspectos biológicos/fisiológi cos (Klon off et al., 1994; Dishman, 1981; Dishman \& Gettman, 1980); gênero ( G a rcia et al., 1995); automotivação para a prática do exercício (Farinatti, 1998); disponibilid ade de tem po (Johnson et al., 1990); condição soci oeconômica (Mon tei ro et al., 1998; Rauds epp \& Viira, 2000); con hecimen to sobre exercício físico e acesso a instalações e espaços adequados à prática de exercício físicos (Sallis et al., 2000; Welk, 1999). É importan te assinalar que a importância relativa de cada um desses fatores na adesão à prática do exercício pode va riar de acordo com o local, a população ou o período de tem po estudado.

No que se refere aos fatores ambientais, embora não sejam muitos os estudos (King et al., 1995), é possível afirmar que o ambien te influ encia a prática da atividade física (Welk, 1999). Porém, como a literatura sobre adesão emprega o termo "ambiente" de forma abrangen te para se referir à influência de fatores externos à pessoa, a produção científica nessa área específica acaba se tornando um po u co difusa no tocante à influência dos fatores ambientais. Pa ra se ter uma idéia, estu dos que tratam da influência do ambi en te na adesão abordam fatores bem diversos que vão des de a influência da família (pais, cônjuges), de colegas e de profissionais de saúde até a influência do clima e do acesso a instalações, passando ainda pela disponibilidade de tempo e de recursos financeiros.

No que con cerne ao ambien te físico, há estudos que apontam uma associação entre a proximidade de instalações privadas e a freqüência na prática de exercícios vigorosos (Sallis et al., 1990). À con clusão sem el han te chegaram Brownell et al. (1980), que identificaram que modestas alterações no ambi en te físico são capazes de influ enciar a atividade física realizada no cotidiano. No estu do em questão, o uso de um cartaz que encorajava o uso da escada em locais públicos, estrategicamente posicio$n$ ado en tre ela e uma escada rolante, resultou no aumento do número de pessoas que optavam pela escada duran te o período de exposição do cartaz. Linenger et al. (1991) prom overam alterações no ambiente físico e social de uma base naval americana. Dentre as adequações do ambien te físico estavam a construção de uma ciclovia e a compra de novos equipamen tos para o exercício. Após um ano, os autores identificarm uma melh ora significativa no tem po do te ste de uma milha e meia.

Em que pesem os poucos estudos sobre a influência do ambiente físico na adesão ao exercício, o exame da literatura indica que o acesso a instalações é, provavelmente, uma variável ambiental que interfere na escolha da pessoa em se exercitar (Sallis et al., 1990). Segundo esses autore $s$, a ri queza na oferta de instalações para a pr á tica do exercício além de servir de estímulo visual, chamando a atenção das pessoas para a questão da prática do exercício, reduz barrei ras físicas e psicoló gicas associadas ao exercício, uma vez que a proximidade do local de exercitação diminui o tem po de deslocamen to da pessoa.

A importância das condições ambientais para a prática de atividades físicas também é compartilhada por mais autores. Para Hunter 
(1994), os espaços ao ar livre obviamen te con tribuem de modo potencial pa ra uma vida ativa, além de constituírem pa rte significa tiva da identidade comunitária. Iverson et al. (1985) afirmam que mudanças no ambiente comunitário permitem ampliar as oportunidades para as pessoas se engaja rem na prática de atividades físicas. Hunter (1994) segue o mesmo raciocínio ao defender a necessidade de as pessoas terem uma maior independência em relação à prática de atividades físicas, no que tange ao uso de rec u rsos próprios, da vizinhança e de agências de la zer e de aptidão física na adoção do estilo de vida próprio. De acordo com King (1994), dadas a abrangência e a complexidade do problema da inativid ade física, a alteração desse quadro requer a suplementação e a expansão de ações nos níveis ambiental, organizacional, in stitucional, social e legislativo. Nesse contexto, intervenções ambi entais e na área de políticas públicas são particularmen te importantes para a promoção da atividade física, porque ambas são el a boradas para influ enciar grandes grupos populacionais.

Considerando toda essa complexidade, as ações adotadas por muitos países em prol da prática regular da atividade física devem ser amplas o suficien te para dar conta do fenômeno da adesão. Porém, é possível afirmar que programas e campanhas de promoção de estilos ativos de vida são as ações que vêm se destacandoden tre as estratégias adotadas pelo poder públ i co.

\section{Programas de promoção da atividade física: a experiência brasileira}

NoBrasil, os programas parecem assumir posição dedestaque, ainda que possa haver diferenças no montante de recursos públ i cos destinados à promoção da atividade física, às pesquisas e ao estabelecimen to de políticas públicas na área. De fato, nos últimos anos, essa preocupação com a saúde pode ser sentida em algumas iniciativas que vêm sendo adotadas pelo poder públ ico.

Em 1986, por exemplo, foi cri ado o Programa Nacional de Educação e Saúde através do Exercício Físico e do Esporte, pelos Mnistérios da Saúde e da Educação e do Desporto, envolven do, respectivamente, suas Coordenadoria de Doenças Crônico-Degenerativas e Secretaria de Educação Física. O programa su r giu com o objetivo de contribuir para o aumento da prática desportiva e de atividades físicas pela população, conscientizando-a sobre a importância da atividade física como fator de saúde e estimulando o desenvolvimen to de hábitos mais saudáveis de vida.

Em 1987/1988, o Programa foi implantado em 14 Estados através de convênios com universidades públicas e a Secretaria de Educação Física e Esportes do MEC. A Política Nacional do Programa foi implementada por uma Comissão In terministerial - Mnistérios da Saú de e da Educação - e por um Comitê Assessor, sempre objetivando a discussão do binômio exercício físico-saúde. Mais recentemente, em 1998, foi instituído o Comitê Técnico Científico de assessoramen to das ações do Programa através da portaria no 3.711/1998 do Ministério da Sa ú de (Brasil, 1998).

A coordenação geral do Programa, que hoje é uma realidade em 27 Estados brasileiros, está sediada na Universidade Estadual de Ca mpinas (Unicamp). Por conta disso, é na Faculdade de Educação Física da Unicamp que está o Núcleo de Educação à Distância em Exercício Físico e Sa ú de que tem a função de orientar e divulgar as ações do programa. Dentre as ações implementadas por esse programa estão a edição de materiais de educação à distância sobre Atividade Física e Saúde, publicados em 1986 (1a edição) e 1996 (2a edição, revista e atualizada, com ti ra gem de 2.000 exemplares).

Além desse su porte e da coordenação geral, há coorden adores em cada Estado da federação formando, assim, uma rede nacional de informações sobre o de s envo lvimento das ações vo ltadas à promoção da prática de atividades físicas. O último en con tro do Programa - III Encon tro Nacional do Programa de Educação e Saúde através do Exercício Físico e do Esporte realizou-se de 6 a 10 de novembro de 2001 na cidade de Salvador. Nesse III Encon tro reiteraram-se os objetivos do programa, den tre os quais está a implementação, em parceria com as universidades federais e estaduais, de estratégias e de equipes locais para o fomen to de programas de atividade física e saúde à população.

Em de zembro de 1996, foi lançado o Programa Agita São Paulo pelo Cen tro de Estudos do Laboratório de Aptidão Física de São Caet ano do Sul (Celafiscs), a tendendo à solicitação da Secretaria de Saúde do Estado de São Paulo de criar um programa para promover saúde por meio da ativid ade física. Os objetivos principais do Agita São Paulo são (a) increm entar o conhecimento da população sobre os benefí- 
cios da atividade física e (b) aumentar o envolvimen to da população com a atividade física. Para isso, o Programa el ege como alvos principais os escolares (crianças e adolescentes), os trabalhadores (colarinho branco e azul) e os i dosos (Celafiscs, 1998).

Para tanto, o Agita São Paulo conta com o a poio do poder públi co (na figura do governo do Estado de São Paulo e de suas Sec retarias de Saú de e da Juventude, Esporte e Lazer), de várias instituições, universid ades, além de nu merosos assessores científicos. Em sua estrutura organizacional, o programa po s sui um colegi ado, composto de várias instituições (governamentais e não-governamentais), que se reúne periodicamen te para definir as ações e estratégias a serem implem en tadas no ano, que se tradu zem notadamente na el a boração de material educativo (cartazes, fôlderes, manuais, slides e vídeos) e na organização de inúmeros eventos, dentre os quais destacam-se o Dia do Escolar Ativo (30 deago s to ), o Dia do Trabalhador Ativo (1o de maio) e o Dia do Idoso Ativo (28 de setem bro) (Celafiscs, 1998). Desde a sua criação, o Agita São Paulo vem promovendo a cap acitação de agen tes de promoção da atividade física (os 'a gen tes Agita') e multiplicando seus programas em várias cidades brasileiras.

A difusão do Programa Agita São Paulo se deu de forma tão expressiva que, em 2001, o poder público federal o escolheu para pautar suas ações estratégicas de promoção da ativi$\mathrm{d}$ ade física em nível nacional. Conheci do como Agita Brasil, o Programa Nacional de Promoção da Atividade Física foi ado t ado pelo Min istério da Saúde (Brasil, 2001) como forma de incrementar o conhecimen to da população sob re os benefícios da atividade física, chamando a a tenção pa ra a sua importância como fator predominante de proteção à saúde, no intuito de envolvê-la na prática de tais atividades (Brasil, 2002). Pa ra tanto, além das crianças, adolescentes, trabalhadores e idosos, o Agita Brasil incluiu os portadores de doenças crônico-degenerativas como um de seus gru pos focais.

A mensagem utilizada pelos Programas "Agita" para a promoção de estilos ativos de vida apóia-se na recom endação adotada por programas dos EUA e, na prática, a s sume o seguinte enunciado: Atividade física é saúde: acumule 30 minutos por dia (...) na maioria dos dias da semana, sepossivel todos, de fo rmacontínua (...) ou em sessões acumuladas de 10, 15 ou 20 minutos (Celafiscs, 1998).

\section{A questão do sedentarismo endêmico}

Em que pesem iniciativas de promoção do exercício em âmbi to educacional, t a lvez a mais difundida na soci ed ade brasileira (seja em congressos científicos, pela mídia ou por meio de ações governamentais) esteja mesmo nos programas em prol de estilos ativos de vida. No entanto, o esforço desses programas em pro$m$ over a prática regular da atividade física parece não estar surtindo os efeitos esperados, uma vez que gran de parte da população brasileira permanece fisicamente inativa. Embora não sejam mu i tos os dados sobre sedentarismo e ainda haja uma certa dificuldade em obtê-los, há pesquisas que nos perm i tem ter um panorama da situação brasileira.

Estu do realizado no final da década de 1980, en comendado pelo Mnistério da Saúde, revelou números bastante escl a recedores acerca da prevalência do seden ta rismo na população brasileira. Das 2.003 pessoas entrevistadas, 33\% declararam praticar exercícios regularmente e som en te $10 \%$ admiti ram fazê-lo com freqüência superior a duas vezes semanais (Brasil, 1988). O principal motivo alegado para a prática do exercício foi a manutenção da saúde e da resistência física.

Em fins da década de 1990, o Insti tuto Brasileiro de Geografia e Estatística (IBGE) em convênio com o Banco Mundial conduziu a Pe s quisa sobre Padrões de Vida (PPV), na qual cerca de 5.000 domicílios em 554 setores nas regiões Nordeste e Sudeste do país foram pesquis ados com o obj etivo de forn ecer informações adequadas para o planeja m en to, acompanhamen to e análises de políticas econômicas e programas sociais em relação aos seus impactos nas condições de vida domiciliar (Brasil, 1999). No que se refere ao exercício físico, o estu do identificou que $19,2 \%$ das pessoas declararam pra ticá-lo semanalmente. Porém, quando considerada freqüência igual ou superior a três ve zes por semana e du ração igual ou maior que 30 minutos, a proporção de declaran tes fisicamente ativos reduziu-se para 7,9\%. Di feren temen te do estudo anterior, quando indagadas sobre os principais motivos para a prática do exercício físico, cerca de 74,5\% das pessoas que declararam praticar exercício semanalmen te apontaram o lazer, a diversão e a estética. Nesse grupo, a penas 10,4\% apon t a ram a saúde, a fisioterapia e o acon sel ha men to médico como motivos para a prática de exercícios físicos. 
Embora de men or abrangência populacional e envolvendo grupos diferentes, há outros estudos que de certo modo corroboram os achados sobre sedenta rismo na população brasileira. Pe s quisa realizada no final da década de 1980 apon tou uma prevalência de sedentarismo de $69,3 \%$ para a população do município de São Paulo (Rego et al., 1990). Mais recen temente, Gomes et al. (2001) identificaram que $59,8 \%$ dos hom ens e $77,8 \%$ das mulheres residen tes no município do Rio de Janei ro com 12 anos ou mais de idade rel a $t$ a ram nunca ter praticado atividade física de lazer. Níveis semelhantes de sedentarismo foram identificados em estudantes. Tomando por base amostra de 325 alu nos, Si lva \& Malina (2000) en con tra ra m alta prevalência de seden tarismo em adolescentes da rede pública de ensino do município de Ni terói (RJ), uma vez que $85 \%$ dos meninos e $94 \%$ das meninas foram con si derados seden tários. Valores mu i to próximos a esses foram encontrados por Gu edes et al. (2001) em alunos do ensino médio do município de Londrina (PR). Cerca de $94 \%$ das moças e $74 \%$ dos rapazes foram con siderados inativos ou moderadamente ativos, o que, segundo os autores, não atende às recomendações quanto à prática de a tivid ade física que possa alcançar impacto satisfatório à saúde.

Por outro lado, há estudos que apontam prevalências de seden ta rismo um po u co menores. Pesquisa realizada no início da década de 1990, com 1.157 indivíduos entre 15 e 64 anos de idade, residentes no município de Porto Al egre, i dentificou uma prevalência de sedentarismo geral de 47\% (Duncan et al., 1993). Mais recen temente, Oehlschlaeger et al. (2004) verificaram prevalência de sedentarismo de $39 \%$ no município de Pelotas (RS), mas com base numa amostra de 960 adolescen tes com $i d$ ades en tre 15 e 18 anos, comu m en te mais ativos que adul to $s$.

Para embaraçar ainda mais a situação, vale citar a pesquisa con duzida pela Fundação Osw a l do Cruz (Fiocruz) / Organização Mundial da Sa ú de (OMS) (Szwarcwald, 2004), cujos resultados preliminares apontam uma prevalência de sedentarismo inferior à en contrada nos estudos anteri ores. No que diz respei to à atividade física (no lazer ou rel acionada ao trabalho), seja vigorosa, moderada ou caminhada, $24 \%$ dos entrevistados relataram praticar menos que 150 minutos por semana, o que é con siderado insuficiente de acordo com os critérios da OMS. O percen tual de pessoas que não realiza nen hum tipo de atividade física é de $11 \%$ na população total, atingin do o patamar de $20 \%$ en tre os indiví duos mais idosos e de men or nível socioeconômico.

Ainda que alguns desses dados não sejam tão recentes e alguns deles não possam ser comparados diretamente, ten do em vista a diferença de metodologias utilizadas, parece-nos razoável admitir que a maioria da população brasileira não pratica atividade física regularmente, o que inclu s ive é confirm ado por vários a utores (Mon tei ro et al. , 2003; Brasil, 2002; Lemos, 2001; Matsu do et al., 2001). No que se refere à motivação para essa prática, porém, os resultados dos estu dos em nível naci onal (Brasil, 1999; 1988) são conflitantes, colocan do em dúvida a idéia, ra zoavel $m$ en te aceita pelo sen so comum, de que a saúde é o principal motivo que leva as pessoas à prática de exercícios.

\section{Discutindo alguns elemen tos para a avaliação de programas}

Pelo expos to até o mom en to, é possível afirmar que programas e campanhas se apre s entam como o que há de mais consisten te no que se refere a ações para a promoção da ativid ade física em nível nacional. Embora haja estudos e propostas para que a Educação Física escolar a s suma como uma de suas funções a prom oção de estilos ativos de vida como forma de combater o seden ta rismo da população bra sil ei rae, por conseguinte, reduzir o índice de doenças crônicas não-transmissíveis (Nahas \& Corbin, 1992; Guedes \& Guedes, 1992; 1993a; 1993b; 1994), elas não chegam a consti tuir um movimen to de escopo nacional e de caráter governamental. Um dos documen tos que poderiam sugerir esse direcionamen to para a Educação Física escolar brasilei ra seria o que estabelece os Parâmetros Curriculares Nacionais (PCN), publicado em 1998 pelo Mnistério da Educação. Os PCN, de fato, ma rcam a íntima relação que os con te ú dos da Educação Física (jogo, e sporte, dança, ginástica e luta) podem ter com o tema transversal "saúde", no entanto, não parecem su gerir que a essa disciplina escolar caberia o papel primeiro de con tri buir para a redução dos índices de sedentarismo da população brasileira.

Não obstante, a Educação Física escolar não fica imune a interferências dos programas próatividade física. O Programa Agita São Paulo, por exemplo, também visa influ enciar a Educa- 
ção Física escolar a promover estilos ativos de vida e a melhorar a qualidade das aulas, aumentando o tempo real de ativid ade física nas mesmas (Celafiscs, 1998). Além disso, são realizados eventos reunindo escolas estaduais, municipais e boa parte das escolas particulares do Estado de São Paulo para discutir com alunos, professores, pais e dirigen tes escolares a atividade física como forma de promoção da saúde (Agita Galera) e distribuídos materiais instrucionais (cartazes, cartilhas etc.) pela Sec retaria de Estado de Educação de São Paulo e pelo próprio programa.

Essas considerações apenas vêm confirmar a preponderância que, ousamos afirmar, os programas têm em relação à totalidade das ações em prol da prática sistemá tica de atividades físicas implementada pelo poder público brasileiro. Ora, con siderando que esses programas são subsidiados em boa parte com recursos públ i cos (Ma t su do et al., 2003), sua permanen te avaliação é fundamental não só para se analisar a pertinência do investimen to públ i co, mas também das ações e estratégias adotadas, tomando-se como referência os objetivos propostos e a correspondência com os resultados obtidos. Em linhas gerais, pode-se dizer que os programas visam ampliar o conhecimen to da população acerca da importância da prática regular de atividades físicas para a saúde e aum entar a parcela da população fisicamen te ativa de modo a con tribuir para a prevenção das doenças crônicas não-transmissíveis. Isso se expressa, por exemplo, nos objetivos do Programa de Educação e Saúde através do Exercício Físico e do Esporte: (a) capacitar profissionais das áreas de Educação, através de metodologia de ensino à distância, em Exercício Físico e Saúde; (b) implementar (...) equipes locais pa ra ofo men to de pro gramas de atividade física e saúde junto à população; (c) produzir material para a população em geral e pa ra os profissionais das áreas de Educação e Saúde sobre a importância da prática da atividade física como fator de promoção de saúde; e (d) implementar o Programa junto às Secretarias Estaduais e Municipais de Saúde e outros (...) como instrumento de promoção e recuperação da saúde, de prevenção das doenças, de melhoria do bem-estar social e da qualidade de vida (Unicamp, 2001).

Objetivos semelhantes também podem ser observados no Agita Brasil, principalmen te no que se refere à preocupação com a difusão de conhecimentos sobre a prática de atividades físicas e o fom ento da adesão a estilos ativos de vida com vistas à redução de doenças crônicas não transmissíveis na população. Senão vejamos: (a) divulgar informações relativas aos benefícios da atividade física e promover o envolvimento da população nestas práticas, enfatizando a sua importância como fator essencial de proteção à saúde, em espe cial no caso de portado res de doenças crônicas não-transmissíveis; (b) promover ações e atividades que induzam as pessoas a adotar, de forma regular, a prática de atividades físicas como um hábi to de vida; (c) constituir-se instrumen to de sustentabilidade e efetividade às ações de prevenção e co n trole de doenças crônicas não-transmissiveis; $e(d)$ desenvolver estudos e formular metodologias nacionais capazes de comprovar benefícios e avaliar impactos da promoção da atividade física na alteração de hábitos de vida e no controle e prevenção das doenças crônicas não-transmissíveis (Brasil, 2001).

Sendo assim, a avaliação do desempenho desses programas deve paut ar-se em indicadores que ex press emo alcance dos obj etivos anteriormente formulados. Nesse sentido, esperam-se estu dos que, tom a n do como referência determinada região atendida por programas de promoção de atividades físicas e respeitando tempo mínimo necessário para se observa rem mudanças, apon tem, por exemplo, o percentual da população fisicamen te ativa, o nível de conhecimen to acerca da importância e da prática regular de atividades físicas propriamente dita e a prevalência de doenças crônicas nãotransmissíveis.

Nesse particular, apesar da escassez de estudos de âmbi to naci onal (ou de larga abrangência populacional) que avaliam iniciativas de promoção da atividade física, $\mathrm{p}$ a rece haver uma certa convergência desses pou cos em apontar que programas e campanhas são efetivos em melhorar o nível de conhecimento da população e suas ati tu des em relação à prática de ativid ades físicas, porém, limitados em ampliar o nível de adesão a essa prática (Simpson et al., 2003; Wardle et al. , 2001; Cavill, 1998; Hill s don et al., 2001). Um dos motivos apres en tados está na dificuldade que programas teriam para alterar o qu ad ro social desfavor á vel à prática reg ular de ativid ades físicas (Cavill, 1998).

Por outro lado, há estudos que apontam progressos no hábito de a população praticar ativid ades físicas regularmente. Na Finlândia, por exem plo, em 25 anos de um programa nacional para redução de fatores de risco para doenças cardiovasculares [North Karelia Project], identificou-se um aumen to no tem po de- 
dicado à prática de atividades físicas no lazer (Puska, 2002). NoCanadá, a prá tica de atividades físicas aumen tou nas décadas de 1980 e 1990 (Craig et al., 2004). Nos EUA, estudo levado a cabo pelo CDC apon tou que o sedentarismo no lazer sof reu redução, embora modesta, no período de 1988 a 2002, especialmen teapós 1996. No Brasil, o programa Agita São Paulo também vem identificando reduções nos nívei s de sedentarismo da população de São Paulo principalmente quan do rel aci on ados ao con hecimen to da mensagem principal do programa (Matsudo et al., 2003; Matsudo et al., 2002a; Matsudo et al. , 2002b). Como vimos, esses result ados são, de certo modo, d iver gentes aos identificados em outros países (Simpson et al., 2003; Wardle et al., 2001; Cavill, 1998; Hills don et al, 2001).

Entretanto, a par da inconsistência desses achados, é grande o reclamo para os elevados níveis de sedentarismo das populações de diferen tes países e a necessidade de mudanças nesse quad ro (CDC, 2004; Craig et al., 2004; Patterson et al., 2004; Brasil, 2002; Lemos, 2001; Brasil, 2001; WHO/CDC, 2000; Bern s tein et al., 1999). Esse cenário nos leva a su por que campanhas e programas em prol da prá tica regular de atividades físicas tenham resultados tímidos. Há, portanto, necessidade de se avaliar mais profundamente tais iniciativas e revê-las, se for o caso, em ben efício de ações mais prof ícuas, consisten tes e duradouras de promoção da ativid ade física na população. Cavill (1998) chega a pon to de argumentar que não se pode esperar que campanhas sejam responsáveis pela reversão de comportamen tos social e ambientalmente co ns truídos.

Em outras palavras, programas e campanhas não teriam condições de alterar o quad ro de seden ta rismo da população se re s tritas a iniciativas de ampliação do con hecimen to da população sobre atividades físicas. Com efeito, há a utores que argumentam que a informação, por si só, é insuficientepara induzir mudanças ou incorporação de hábi tos de vida (Dishman et al., 1985; Sieden top, 1996). Sen do assim, ações que se con cen trem em modificar os fatores de riscoem indivíduos, sem levar em con sideração o con texto social em que vivem, dificilmente prom overiam melh oras significativas (Si eden top, 1996).

Nesse sentido, é problemático ad mi tir que a avaliação de programas, como o Agita Brasil por exemplo, possa se limitar a quantificar e analisar as atividades e ações desenvolvidas nos níveis lo- cais de estímulo à atividade física e a quantificar e analisar a inco rpo ração do conhecimen to da população sob re os benefícios da prá tica de atividade física, que residem nas cidades onde o Programa foi implantado (Brasil, 2001). Ora, se um dos objetivos finais do programa é levar as pessoas a adotar a prática de atividades físicas como um hábi to de vida, há que se ter indicadores que permitam avaliar até que pon to esse obj etivo foi alcançad. Sem dúvida que a men su ração do nível de atividade física da população deve ser um dos indicadores a serem con s i derados na avaliação de programas cujos obj etivos são ampliar a adesão à ativid ade física.

\section{Considerações finais}

Queremos trazer para o debate, entretanto, a possibilidade de se considera rem outros indicadores para a avaliação desses programas, que expressem outras variá veis intervenientes no processo de adesão à prática de atividades física. Tal con si deração é pertin en te uma vez que, como foi vis to também é objetivo desses programas criar condições favoráveis e promover ações (...) que induzam as pessoas a adotar, de forma regular, a prática de atividades físicas como um hábi to de vida (Brasil, 2001).

Considerando que a adesão é um fenômeno complexo, e que deve ser enten dido como um process o, o hábi to de praticar atividades físicas ex pressa, a rigor, o comportamento final almejado. Assim sen do, ter em conta esse comportamento como o único indicador da efetividade de programas e de iniciativas de promoção de atividades físicas seria enfatizar o produto e de sconsiderar o processo, o que, a nosso ver, não seria justo. Isso significa dizer que é possível que a maioria de determinada população não pratique atividades físicas regularmente, mas, ao mesmo tem po, condições favor á veis à sua prática tenham sido criadas por programas ou outras iniciativas do poder públ i co. Em outras palavras, seria plausível afirmar que, apesar de não ter ocorri do uma redução sign ificativa na prevalência de sedentarismo da população de determinada região, pode ter havido uma mel h oria na possibilidadede seus membros aderi rem à prática de atividades físicas, expressa, por exemplo, numa maior oferta de espaços públ i cos desti n ados ao lazer ativo. Portanto, a identificação e avaliação dessas condições (leiam-se va riá veis) aju da riam a expressar, por exemplo, o potencial de adesão à prática de 
ativid ades físicas em determinado contex to e, em última análise, a eficácia (em sentido lato) de programas e de iniciativas em prol da atividade física.

Iniciativa nesse senti do foi con duzida pela Prefei tu ra da cidade de Belo Hori zon te. Com o objetivo de mon i torar os impactos das ações e in tervenções públicas para o cidadão e perm itir uma distribuição mais eficien te e justa dos recursos públicos municipais, criou-se um Índice para a Qualidade de Vida do Lugar Urbano (IQVU) com base no acesso à oferta de bens e serviços (Prefeitura de Belo Horizonte, 2001). Para tanto, definiram-se on ze variáveis (Abastecimento; Assistência Social; Educação; Esportes; Cultura; Habitação; Infra-estrutura Urbana; Meio Ambiente; Saúde; Serviços Urbanos; Seg u rança Urbana) que foram divididas em componentes que, por sua vez, produziram indicadores para expressar numericamen te a quantidade eaqualidade da oferta dos serviços pesquisados em cada região.

Uma dessas variáveis, definida como "Esportes", teve como compon en tes "Equipamentos Esportivo s", avaliados pela "área por habitante de quadras, piscinas, campos, clubes e congêneres", e "Promoções Esportiva s", analisadas com base no "número de eventos esportivos e freqüência de públ i co" (Quad ro 2). Em bora a definição dessa va riá vel tivesse por trás a intenção de avaliar ações do poder público na área de esportes e lazer sem uma preocupação imediata de inferir o po tencial de determinada região de favorecer a prática de atividades físicas, esse é um bom exemplo de como outras variáveis podem ser incluídas na avaliação de programas e campanhas em prol da atividade física.

Acrescen te-se a isso o fato de a prática regular de atividades físicas ser um comporta- mento complexo (bem diferente do uso habitual do cinto de segurança, por exemplo). Em mu i tos casos, a adesão é efêmera; há aumen to na prática de atividades físicas qua ndo há estímulos para tal sendo constantemente veiculados. Uma vez retirados, a adesão ten de a voltar aos níveis iniciais (Brownell et al., 1980). Sendo assim, a alteração da prevalência do sedentarismo requ er ações nos níveis ambiental, organizacional, institucional, social e legislativo.

Essas con clusões poderiam ser estendidas a todo e qualqu er com pon en te dos modos de vida. As influências ambi entais, portanto, devem ser analisadas sob uma pers pectiva que ex tra pole a dimensão da estrutura física, depen den do também do en torno social. A prob abilidade de que a ativid ade física passe a fazer parte da rotina de um grupo, por exemplo, parece-nos aumentada se forem realizadas mudanças no entorno social, desde o de senvo lvimen to de uma consciência coletiva no que toca ao reconhecimento de sua importância até alterações nas relações en tre tempo de trabalho e lazer, o que implica repensar fatores como jorn ada de trabalho, rendimentos, sistema de transporte público, oferta, distri buição e acessibilidade de equipamentos desportivos e espaços públicos para a prática de ativi d ades físicas, den tre outros.

A nosso ver, a avaliação de programas próatividade física poderia ser feita com base na análise de um número maior de variáveis, contemplando melhor o fenômeno da adesão. A definição de outras variáveis, emanadas de uma exaustiva revisão de literatu ra e da realização de estudos empíricos, se apresenta como um passo importante para uma avaliação mais consisten te e fidedigna de programas de promoção da atividade física de larga abrangência populacional.

Quadro 2

Componentes e indicadores da variável Esportes.

\begin{tabular}{lll}
\hline Variável & Componentes & Indicadores \\
\hline Esportes & Equipamentos esportivos & $\begin{array}{l}\text { Á rea por habitan te de quadras, piscinas, } \\
\text { campos, clu bes e congêneres }\end{array}$ \\
Promoções esportivas & $\begin{array}{l}\text { Número de even tos esportivos e freqüência } \\
\text { de públ i co }\end{array}$ \\
\hline
\end{tabular}




\section{Colaboradores}

MS Ferrei ra trabalhou na con cepçãoteórica, el a boração e redação final do tex to e AL Najar parti c i pou da discussão teórica e trabalhou na revisão e organização do texto.

\section{Referências bibliográficas}

Andersen LB, Schnohr P, Schroll M \& Hein, HO 2000. Allcause mort a $\mathrm{l}$ ty assoc $\mathrm{i}$ a ted with ph ysical activi ty du ring leisu re time, work, sports and cycling to work. Archives of Internal Medicine 160:1621-1628.

An d rewGM et al. 1981. Reasons for Dropo ut from exercise Programmes in Post-Coronary Pa ti ents. Medicine and Science in Sports and Exercise 13(3):164-168.

Armstrong $\mathrm{N}$ et al. 1990 . Health-related physical activity in the nati onal curriculum. British Jou rnal of Physical Education 21: 225.

Bern stein MS, Mora bia A \& Sloutskis D 1999. Definition and prevalen ce of sedentarism in an urban population. American Jou rnal of Pu blic Health 89(6):862-867.

Bid dle S 1987. Exercise motivation: theory and practice, pp. 139-144. In S Biddle (ed.). Health-rel a ted fitness in physical education. Ling Publishing House, Lon don.

Blair SN et al. 1995. Changes in physical fitness and allcause mortality: a prospective study of healthy and unhealthymen. The Jou rnal of the American Medical Association 273:1093-1098.

Blair SN et al. 1989. Physical fitness and all-cause mortality: a prospective study of healthy men and women. The Journal of the American Medical Association 262:2395-2405.

Brasil 2002. Ministério da Saúde. Sec retaria de Políticas de Saúde. Revista de Saúde Pública 36(2):254-256.

Brasil 2001. Mnistério da Sa ú de. Portaria no 1.893/GM, de 15 de outu bro de 2001. In stitui o Programa de Promoção da Atividade Física. Disponível em <http:// dtr2001.saude.gov.br/portarias/2001/i\%201893\% 20PROGRAMA\%20DE\%20ATIVIDADE\%20FISI CA\%20PELA\%20SPS.rtf $>$. Acesso em 22 set. 2004.

Brasil 1999. Pesquisa sob re padrões de vida 1996-1997. I BGE, Rio de Janeiro.

Brasil 1998. Min istérioda Saúde. Porta ria no 3711/GM, de 7 de outu bro de 1998. In stituio Comitê Técnico Científico - CTC, de Assessoramento às Ações de Atividade Física e Saúde. Disponível em <http:// www. saú de.gov.br/portarias/1998.htm>. Acesso em 8 set. 2003.

Brasil 1988. Mnistério da Saúde. Divisão Nacional de Doenças Crôn i co-Degen era tivas. Projeto Saúde: estudo sobre estilo de vida. LPM [Levantamentos e Pesquisas de Marketing Ltda.], São Paulo.

Brownell KD, Stunkard AJ \& Albaum JM 1980. Evaluation and modification of exercise patterns in the natural environment. American Journal of Psychiatry 137(12): 1540-1545.

Bull FC \& Jam rozik K 1998. Advice on exercise from a family physician can help sedentary patients to become active. American Jou rnal of Preventive Medicine 15(2):85-94.

Carvalho YM 1995. O 'mito' da atividade física e saúde. Hucitec, São Paulo.

Cavill N 1998. Nati onal Campaigns to PromotePhysical
Activity: can they make differen ce? In tern a tional Jou $r$ nal of Obesity 22(2): S48-51.

CDC 2004. Preval en ce of no leisu re-time physical activity - 35 states and the District of Columbia, 1988-2002. MMWR Morbidity and Mortality Weekly Report 53(4):82-86.

CDC 2000. Pro moting Physical Activity: a best buy in Pu blic Health. CDC, Atlanta.

Celafiscs 1998. Programa Agita São Paulo. Celafiscs, São Paulo.

Craig CL, Russell SJ, Cameron C \& Bauman A 2004. Twen ty - year trends in physical activity among Ca n adian Adults. Canadian Journal of Public Health 95(1):59-63.

Dishman RK (ed.) 1994. Advances in exercise adherence. Human Kinetics, Champaing.

Dishman RK 1981. Biologic influ en ces on exercise ad h erence. Re se a rch Quarterly for Exerci se and Sport 52(2): 143-159.

Dishman RK \& Get tman LR 1980. Psychobiologic influences on exercise adherence. Journal of Sport Psych ology 2: 295-310.

Dishman RK, Sallis JF \& Orenstein DR 1985. The determinants of physical activity and exercise. Public Health Reports 100(2):158-171.

Duncan BB et al. 1993. Fatores de risco para doenças nãotransmissíveis em área metropolitana na região Sul do país: prevalência e simultaneidade. Revista de Saúde Pública 27(1):143-148.

Farinatti PTV 2004. Envelhecimen to, promoção da saúde e exercício: bases teóricas e metodológicas. Ed. Manole, São Paulo (no prelo).

Farin at tiPTV 1998. Mi se au point d'une te chnique d'evaluation de l'autonomie d'action des senio rs: de la theorie à la pratique. Tese de doutorado. In si tut Su periéur d'Education Physique et Kinesithérapie-Université Libre de Bruxelles, Bruxelles.

Garcia AW et al 1995. Gender and devel opm ental differen ces in exercise bel $\mathrm{i}$ efs among youth and pred $\mathrm{i}$ cti on of their exercise beh avior. Journal of School Health 65(6):213-219.

Gomes VB, Siqueira KS \& Sichieri R 2001. Atividade física em uma amostra probabilística da população do município do Rio de Janei ro. Cad ernos de Saúde Pública 17(4):969-976.

Gu edes DP, Gu edes JERP, Ba rbosa DS \& Ol ivei ra JA 2001. Níveis de prática de ativi d ade física habi tual em adolescentes. Revista Brasileira de Medicina do Esporte 7(6):187-199.

Gu edes DP \& Gu edes JERP 1992. Proj eto Ativi d ade Física eSaúde: uma proposta de promoção de saúde. Revista da APEF - Londrina 7(13):15-22.

Gu edes DP \& Gu edes JERP 1993a. Subsídios para imple$\mathrm{m}$ entação de programas direcion ados à promoção da 
saúde através da educação física escolar. Revista da APEF - Londrina 8(15):3-11.

Guedes DP \& Guedes JERP 1993b. Educação física escolar: uma proposta de promoção da saú de. Revista da APEF - Londrina 7(14):16-23.

Guedes DP \& Guedes JERP 1994. Su gestões de con teúdo programáti co para programas de edu cação física escolar direcionados à promoção da saúde. Revista da APEF - Londrina 9(16):3-14.

Ha skell WL, Mon toye HJ \& Orenstein D 1985. Physical activity and exercise to achi eve health-rel a ted phys ical fitness com ponents. Public Health Reports 100(2): 202-212.

Hills don M, Cavill N, Na n chahal K, Di a m ond A \& White IR 2001. National level prom o tion of physical activity: results from England's Active for Life campaign. Journal of Epidemiology and Community Health 55:755-761.

Hunter D 1994. Community determinants and benefits of active living, pp. 203-206. In HA Quinney, L Gauvin \& AET Wall (eds.). Toward active living: proceedings of the intern a tional co nference on physical activity, fitness and health. Human Kinetics, Champaign.

Ivers on DC, Fielding JE, Crow RS \& Christen son GM 1985. The prom o ti on of physical activity in the United States population: the status of programs in medical, worksite, com mu nity and sch ool set tings. Public Health Reports 100(2):212-224.

Johnson CA, Corrigan SA, Dubbert PM \& Gramling SE 1990. Perceived barriers to exercise and wei ght control practices in community women. Women \& Health 16(3/4):177-191.

King AC et al. 1995. Envi ron mental and policy approaches to cardiovascular disease prevention through physical activity: issues and opportunities. Health Education Quarterly 22(4):499-511.

King AC 1994. Com mu n ity and public health approach es to the promotion of physical activity. Medicine and science in sports and exercise 26(11):1405-1412.

King AC et al. 1992. Determinants of physical activity and interventions in adults. Medicine and Science in Sports and Exercise 24(6):S221-236.

Kl on off EA, An nechild A \& Landrine H 1994. Predicting exercise ad heren ce in women: the role of psychologi$\mathrm{cal}$ and physiological factors. Preventive Medicine 23:257-262.

Lee IM, H shiehCC \& Paffenbarger RS 1995. Exercise intensity and longevity in men - the Ha rvard Alumni Health Study. The Journal of the American Medical Association 273(15):1179-1184.

Lee IM, Paffenbarger RS \& Hen nekens CH 1997. Physical activity, physical fitness and longevity. Aging 9(1-2):2-11.

Lemos MSA 2001. Reduzin doo sedentarismo nos cen tros urbanos. Revista Promocãa da Saúde 2(5):9-10.

Lin en ger JM, Chess onCV \& Nice DS 1991. Physical fitness gains foll owing simple environ m ental change. American Jou rnal of Preven tive Medicine 7(5):298-310.

Matsu do SMM et al. 2003. The Agita São Paulo Program as a model for using physical activity to promote health. Revista Panamericana de Saúde Pública 14(4): 265-272.

Matsudo SMM et al. 2002a. Nível de ativid ade física da população do Estado de São Paulo: análise de acordo com o gênero, idade, nível socioeconômico, distribuição geográfica e de conhecimento. Revista Brasilei ra de Ciência e Movimento 10(4):41-50.

Matsu doV et al. 2002b. Promotion of physical activity in a developing country: the Agita São Paulo experience. Public Health Nutrition 5(1A):253-261.

Matsu do SMM et al. 2001. Promoção da saúde mediante o aumen to do nível de atividade física: a proposta do programa Agita São Paulo. Revista Âmbi to Medicina Esportiva 7:5-15.

Meeusen R \& Borms J 1992. Gymnastic injuries. Sports Medicine 13(5):337-356.

Meinberg E 1989. Para uma nova ética do desporto, pp. 69-76. In JO Bento \& A Marques (eds.). Desporto, Ética, Sociedade. Editora da FCDEF, Porto.

Montei ro CA et al. 2003. A descriptive epidemiology of leisure-time physical activity in Brazil, 1996-1997. Revista Pa namericana de Salud Publica/Pan American Journal of Public Health 14(4):246-254.

Monteiro HL, Gonçalves A, Padovani CR \& Fermino Neto JL 1998. Fa tores socioecon ô $\mathrm{mi}$ cose ocupacionais e a prá tica de atividade física regular. estudo a partir de policiais militares em Bauru. São Paulo. Revi sta Motriz 4(2):91-97.

Morris JN, Clayton DG, Everitt MG, Semmence AM \& Bu r gess EH 1990. Exercise in leisu re time: coronary attack and death rates. British Heart Journal 63(6): 325-334.

Morris JN, Everitt MG, Pollard R, Ch ave SPW, \& Semmen ce AM 1980. Vigorous exercise in leisure time: protection against coronary heart disease. Lancet ii: $1207-1210$.

Morris JN, He ady JA, Raffle PAB, Roberts CG \& Pa rks JW 1953. Coron a ry heart disease and physical activity of work. Lancet ii:1053-1057.

Nahas MV \& Corbin C 1992. E ducação para a ap tidão física e saúde: justificativa e su gestões para implem entação nos programas de educação física. Revi sta Brasileira de Ciência e Movimento 6(3):14-24.

Oehlschlaeger MHK, Pinhei ro RT, Horta B, Gelatti C \& Sa n t' Ana P 2004. Prevalência e fatores associados ao s eden $t$ a rismo em ado le s cen tes de área ur bana. Revista de Saúde Pública 38(2):157-163.

Oguma Y, Sesso HD, Paffenbarger RS \& Lee IM 2002. Physical activity and all cause mortality in women: a review of the evidence. British Journal of Sports Medicine 36(3):162-172.

Paffenbarger RS \& Lee IM 1996. P hysical activity and fitness for health and lon gevity. Research Quarterly for Exercise and Sport 67(3):S11-28.

Paffenbarger RS, Hyde RT, Wing AL \& Hsieh CC 1986. Physical activity, all-cause mort alityandlon gevity of co ll ege al umni. The New En gland Jou rnal of Medicine 314(10):605-613

Paffenbarger RS \& Hale WE 1975 . Work activity and coronary heart mortality. The New England Journal of Medicine 292(11):545-550.

Paffenbarger RS, Laughlin ME, Gime AS, Bl ack RA 1970. Work activity of longshoremen as related to death from CHD and stroke. The New England Journal of Medicine 282(20):1109-1114

Patters on PD, Moore CG, Probst JC, Shinogle JA 2004. Obesity and physical inactivity in rural America. The Journal of Rural Health 20(2):151-159.

Prefei tu ra de Belo Horizonte 2001. Índice de qualidade de vida urbana. Disponível em <http://www.pbh.gov.br/ smpl/iqvu/>. Acesso em 18 ago. 2001.

Puska P 2002. Successful prevention on non-communica- 
ble diseases: 25 year experien ces with North Karelia Project in Finland. Public Health Medicine 4(1):5-7.

Raudsepp L \& Vii ra R 2000. Sociocultural correlates of physical activi ty in adolescents. Pediatric Exerci se Science 12:51-60.

Rego RA et al. 1990. Fa tores de risco para doenças crônicas não-transmissíveis: inquéri to domiciliar no município de São Paulo, SP (Brasil). Metodologia e resultados preliminares. Revista de Saúde Pública 24(4): 277-285.

Sa llis JF, Prochaska JJ \& Taylor WC 2000. A revi ew of correlates of physical activity of dhildren and adolescents. Medicine and Science in Sports and Exercise 32(5):963-975.

Sallis JF \& McKenzie TL 1991. Physical education's role in public health. Research Quarterly for Exercise and Sport 62(2):124-137.

Sa llis JF et al. 1990. Distance bet ween homes and exercise facilities related to frequency of exercise among San Di ego residents. Pu blic Health Repo rts 105(2):179-185.

Sallis JF et al. 1989. A mul tivariate stu dy of determinants of vigorous exercise in a com mu nitysample. Preventive Medicine 18(1):20-34.

Si eden top D 1996. Va luing the physically active life: contemporary and future directions. Quest 48:266-274.

Silva RCR \& Malina RM 2000. Nível de atividade física em adolescentes do município de Niterói, Rio de Janeiro, Brasil. Cadernos de Saúde Pública 16(4):1091-1097.

Simpson ME et al. 2003. Walking trends among U.S. Adults: the beh avi oral risk factor su rveillance system, 19872000. American Journal of Preven tive Medicine 25(2): 95-100.

Sobral F 1990. Investigação das relações entre saúde e desporto: história, estadb atual e pers pectivas de evolução, pp. 41-52. In JO Ben to \& A Ma rques (eds.). Desporto, saúde, bem-estar. Editora da FCDEF, Porto.

Solom onHA 1991. O mito do exercício. Ed. Summus, São Paulo.

Szwarcwald CL et al. 2004. O Brasil em números: pesquisa mundial de saúde 2003. RADIS: comunicação em saúde (23):14-33.

Unicamp 2001. Programa de Educação e Saúde através do Exercício Físico e do Esporte. Dispon ível em $<$ http:// www.ead.unicamp.br/fef/programa/o_programa. html>. Acesso em 10 dez. 2004.

Wardle J, Rapoport L, Miles A, Afuape T \& Duman M 2001. Mass education for obesity prevention: the penetration of the BBC's 'fighting fat, fighting fit' campaign. Health Education Research 16(3):343-355.

Welk GJ 1999. The youth physical activity promotion model: a conceptual bridge between theory and practice. Quest 51(1):5-23.

WHO/CDC 2000. Promoting physical activity: a best buy in public health. CDC Collaborating Center on Physical Activity and Health Promotion, Atlanta.

Wi lliams PT 2003. The illusion of i m proved physical fitness and reduced mortality. Medicine \& Science in Sports \& Exercise 35(5):736-740.

Artigo apres en tado em 22/12/2004

Aprovado em 4/03/2005

Versão final apres en tada em 3/06/2005 